\title{
A feasibility study of PLC technology for digital inclusion
}

\author{
Jorge A. M. de Souza, Marcelino S. da Silva, Carlos R. L. Francês, João C. W. A. Costa, \\ E. V. Segatto, Flavio R. Antonio and Nandamudi. L. Vijaykumar
}

Marcelo

\begin{abstract}
In the current national scene, many actions point at projects of digital inclusion and citizenship. In this context, providing access technologies as a requisite for the implementation of these actions is primordial. In this way, many innovative experiences have been presented in the past few years. This paper presents a study on the PowerLine Communication - PLC technology; as a proposal for a feasible access network for Brazilian Amazon. First, the characteristics of the PLC technology are studied from an implanted indoor prototype at Federal University of Pará. The measures used in this prototype serve as input for a created model, from which it is intended to study the system more widely, considering factors such as: scalability, reliability and the physical characteristics.
\end{abstract}

Index Terms - Computer network performance, powerline communication, access technologies, digital inclusion.

\section{INTRODUCTION}

$\mathrm{W}$ HEN the focus in the use of an access technology is the digital/social inclusion, some points must be previously observed, such as: involved capillarity of the network, involved costs for the final user; and in the process of implantation, reach and interoperability with other already consolidated technologies. In this direction, some access technologies have been researched in the past few years. However, when the basic concern is lack of infrastructure - a reality in the major part of the Brazilian territory, the solution, usually, must be distinguished, once that it is unlikely that an end-to-end communication solution applicable in all the situations is available. In the Amazonian scenario, these particularities are enhanced, given the "difficulties" imposed by the natural characteristics of the region (immense distances, lands discontinued by rivers, great density of the forests, etc). Thus, many studies distinguish the analysis considering the "sui generis" characteristics, as in [4], [3] and [10].

Manuscript received April 15, 2006. This work was supported in part by Eletronorte and $\mathrm{CNPq}$.

J. A. M. S. is with the High Performance Networks Planning Laboratory, Federal University of Pará, Brazil (e-mail: jamsouza@ufpa.br).

M. S. S. is with the High Performance Networks Planning Laboratory, Federal University of Pará, Brazil (e-mail: marcelino@ufpa.br).

C. R. L. F. is with the High Performance Networks Planning Laboratory, Federal University of Pará, Brazil (e-mail: rfrances@ufpa.br).

J. C. W. A. C. is with the High Performance Networks Planning Laboratory , Federal University of Pará, Brazil (e-mail: jweyl@ufpa.br).

M. E. V. S. is with the Electrical Engineering Department, Federal University of Espírito Santo, Brazil (e-mail: segatto@ele.ufes.br).

F. R. A. is with the Eletronorte, Brazil (flavioroberto@eln.gov.br).

N. L. V. is with Laboratory of Computing and Applied Mathematics, Brazilian Institute of Space Research, Brazil (vijay@lac.inpe.br).
The Laboratory of High Performance Networks Planning - LPRAD, of the Federal University of Pará (UFPA), has been developing researches in the area of access technologies (e.g. Digital Subscriber Line - DSL and PowerLine Communication - PLC) with an approach strongly directed to the peculiar characteristics of the Amazon region, toward the digital inclusion, as [2] and [9].

In this context, the PLC technology stands out, given the great capillarity of the power system, exactly in regions with little telecommunications infrastructure. For example, in the state of Pará around 1.156.445 homes are supplied by the power distribution network ${ }^{1}$. This capillarity contrasts significantly when compared to the numbers of the year 2000 Census $^{2}$, which shows that in Brazil there are 17.774.403 homes with a telephonic line installed. In the North region, there are around 821.280 homes with regular telephone. For the state of Pará, there are 350.423 homes with a telephonic line. Thus, in Pará, there are more residences supplied by the power network than homes with a telephonic line in all the of North region, in its seven states. This scene tends to increase with the program in progress "light for all", an initiative of the federal government which counts on the partnership of the state governments and the power suppliers, whose intention is to take electricity to more than the 12 million people, in all the national territory, until the year of 2008, with investments esteemed in $\mathrm{R} \$ 7$ billion.

The presented numbers indicate an important point of reflection: the wideness of the transmission and distribution networks of the electric sector within the regions which traditionally lack an adequate telecommunications infrastructure. This way, systems such as PLC can be used as an alternative access technology, once it is in compliance with the Amazonian reality.

Given the presented motivation, a PLC prototype is being implanted, in Belém, connecting the Eletronorte (ELN) to the Federal University of Pará (UFPA). This prototype will make possible to study the behavior of a PLC communication, exposed to the real conditions of a typical city of the Amazon. Additionally, an indoor PLC prototype is in use, which seeks to reproduce the conditions of a user internal access network.

This paper presents the implantation experience of the mentioned indoor prototype and discourses concerning the

\footnotetext{
${ }^{1}$ Source: Centrais Elétricas do Pará S. A. - CELPA (www.gruporede.com.br/celpa)

2 Source: Instituto Brasileiro de Geografia e Estatística - IBGE (www.ibge.gov.br)
} 
models for study of the basic PLC networks characteristics.

\section{POWERLINe COMMUNICATION}

The PLC technology uses the existing electric infrastructure to provide the data communication. This technology has shown itself sufficiently competitive in the broadband access market, disputing with other more traditional, like DSL. PLC counts with the advantage of having a sufficiently wide infrastructure and with relatively little cost associated [8].

PLC, however, as well as other data communication technologies, possesses some inconveniences. The physical environment is very hostile for the data, once that it was not conceived for this purpose, thus there are many properties of the power systems which influence negatively the high speed communications (losses in the cable, propagation in multiple paths and the noise, for example) [12].

A way to reduce the impact of the transmission environment in the communication is the application of efficient modulation methods, such as OFDM (Ortogonal Frequency Division Multiplexing), besides mechanisms for error correction such as FEC (Forward Error Correction) and the ARQ (Automatic Repeat reQuest) [6].

However, independent of the adopted techniques, a factor to be observed and understood is the noise in the communication.

\section{A. Noise Characteristics}

Given the physical characteristics of the conductor and the nature of the electric energy, this environment is not best indicated for data communication. The propagation of the signal through the power transmission line provokes an attenuation and a delay in the signal, which increases with the distance and the frequency. This attenuation depends on the impedance characteristic (ZL) and on the propagation constant $(\gamma)$ of the transmission lines. In accordance with [13] and [1], these two parameters are resistance functions $\mathrm{R}$, conductance $\mathrm{G}$, inductance $\mathrm{L}$, and capacitance $\mathrm{C}$, per unit of length, which depend on the frequency.

Based on the studies presented in [5] and [13], two types of noises can be adopted: (a) deep noise, formed by deep colored noises, short band noises and asynchronous periodic impulsive noises in relation to the channel frequency; and (b) impulsive noise, formed by asynchronous impulsive noises and synchronous periodic impulsive noises in relation to the channel frequency. Having the latter a great probability to cause bit errors or in bits sequence in a of high speed data communication [12]. The characteristic equations of the channel and noise are presented in subsection B. Besides the inconveniences of the environment, other "obstacles" for the PLC use exist in what refers to the internet access. The subscriber of this service has an interface with a low voltage network, which possess innumerable transformer units throughout its extension. The PLC signal must make a bypass in these equipment, in order to keep the integrity of the information. It can be used, for example, PLC repeaters to avoid the transformer units along the way, typically in low voltage networks. However, depending on the extension of the network, the investment in the implantation of the service can increase substantially for account of this inconvenience.

With the purpose to characterize the typical traffic, as well as the essential characteristics of this type of communication regarding the communication channel and the noises, a PLC prototype, which will be described in section 3 , is being tested.

\section{B. The PLC transmission channel}

As mentioned previously, the characteristics of the power transmission lines depend on the characteristic impedance (ZL) and on the propagation constant $(\gamma)$ of the transmission lines, which are functions of the resistance $R(f)$, conductance $G(f)$, inductance $L(f)$, and capacitance $C(f)$, per unit of length:

$$
\begin{aligned}
& Z_{L}(f)=\sqrt{\frac{R(f)+j 2 \pi \cdot L(f)}{G(f)+j 2 \pi \cdot C(f)}} \\
& \gamma(f)=\sqrt{(R(f)+j 2 \pi \cdot L(f)) \cdot(G(f)+j 2 \pi \cdot C(f))}
\end{aligned}
$$

The transference function of the line in function of the frequency and the distance can be formulated as:

$$
H(f, l)=\exp (-\gamma(f) l)
$$

On many analysis and measurements [13], it was concluded that for high frequencies, such as the PLC frequency band, $1 \mathrm{MHz}$ to $30 \mathrm{MHz}$, the impedance characteristic and the propagation constant can be approached by:

$$
\begin{aligned}
& Z_{L}(f)=\sqrt{\frac{L(f)}{C(f)}} \\
& \gamma(f)=\frac{1}{2} \cdot \frac{R(f)}{Z_{L}}+\frac{1}{2} \cdot G(f) Z_{L}+j 2 \pi f \sqrt{L(f) C(f)}
\end{aligned}
$$

And the transference function can be given by:

$$
H(f)=A(f, l) \exp (-j 2 \pi f \tau)
$$

Where the term $A(f, 1)$ represents the attenuation of the signal and $\exp (-\mathrm{j} 2 \pi \mathrm{f} \tau)$ represents the delay in the signal propagation.

\section{PLC channel model}

The topology of the power distribution networks considerably differs from the traditional communication networks, such as twisted pair, coaxial cable or optic fiber. Numerous reflections of the signal are received and occur mainly due the junction of cables of different impedances. This way, the transference function becomes: 


$$
H(f)=\sum_{i=1}^{N} A\left(f, l_{i}\right) \exp \left(-j 2 \pi f \tau_{i}\right)
$$

Analyzing the time domain, the PLC channel shows the form:

$$
h(t)=\sum_{i=1}^{N} A_{i} \delta\left(t-\tau_{i}\right)
$$

Where $\mathrm{Ai}$ and $\tau \mathrm{i} \mathrm{Ai}$ are the attenuation and the delay of the multiple received signals, and $\mathrm{N}$ is the number of multipaths that considerably affect the channel response.

The PLC channel has the characteristic of being selective to the frequency, this if due to the multipath characteristic of the power transmission networks.

Given the hostile characteristics of PLC channel (multipath, selective to the frequency and the impulsive noise) it is necessary to apply an efficient modulation technique.

For allowing the transmission of data through many independent sub-carriers, the OFDM modulation technique has been a good alternative for the data transmission on the PLC channel. Once that only a small percentage of subcarriers will be reached by the effect of frequency selectivity of the canal, error correction codes can be used to correct the data of the few wrong sub-carriers, as well as new subcarriers can be used to substitute the wrong ones.

The OFDM modulation characteristics regarding the impulsive noise and the multipath are presented in details in [7].

\section{PLC PROTOTYPE}

The PLC Indoor (PLC/LPRAD) archetype is located in the dependences of the Laboratory of Electric Engineering and Computing - LEEC of the UFPA, in which is inserted the Laboratory of High Performance Networks Planning LPRAD - and has the purpose of supplying information regarding the nature of the noise (physical layer), supply typical information the superior layers, load characterization and performance measures of the system (e.g. trustworthiness and scalability). The information obtained in this prototype is serving as entry parameters for the simulations of PLC network in the NS-2 [11].

Besides the attainment of the performance measures, the prototype was used to validate the simulation model, that is, a model in the same scale of the real system is elaborated. Afterwards, the set of performance measures obtained from the simulation with the corresponding measures of the prototype are compared. Having a correspondence with both results, the model can be considered validated and can be studied in scale, with other many scenarios.

\section{A. Topology}

The power house of the LEEC, after the transforming exit from average to low voltage, possess two circuits, one for the building illumination, feeding of the air conditioners and for some outlets in building walls, and the other, for the power keys located in the building rooms, from which each room mounts its internal power system.
In this power house a PLC base station was installed (HE), which is connected to the ethernet network of the LPRAD and to the three phases of the circuit that feeds the power keys of the rooms; this way, all the rooms of the LEEC will possess internet connectivity points through the PLC network. This circuit was chosen given the fact of being wider than the first one.

A gateway/repeater was installed (HG) in the LPRAD, which receives the PLC signal through an outlet fed by the power key of the room, and injects it in an outlet in the wall of the first circuit. With this, we obtain connectivity in all of the outlets within the LPRAD. With this, two PLC modems were connected, one in the first circuit and the other in the second circuit, and a computer in each one of them.

The tests are made through a video transmission between the computers, through the PLC network, thus using the two power distribution circuits of the LEEC. Figure 1 shows the PLC Indoor prototype.

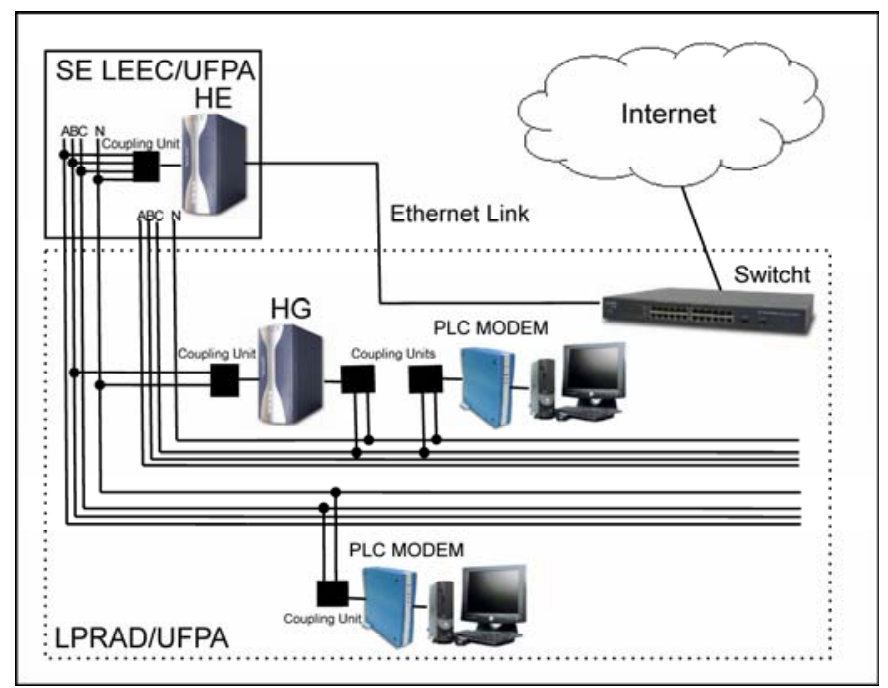

Fig. 1.PLC/LPRAD Prototype Topology

The idea is to be able to impute a series of applications in the prototype, whose loads generate typical and atypical situations; so that we can have a standard for the system behavior given determined applications. Moreover, to observe some specific characteristics of PLC systems, such as noise in the channel, interference and induction inside in the system (e.g. the effect of cellular "rechargers" in the throughput of the system).

\section{MODEL}

The analysis of an indoor PLC access network can be made considering information such as: packages loss, throughput, blocking probability, delay and jitter. This way, the viability of the network for the desired applications can be verified.

The environment described in Section 3 was submitted to a characteristic traffic of a continuous video transmission, application whose QoS requirements are sufficiently rigid. This way, the analysis could be made based on information collected from the worse case. The system was monitored in 24 hour intervals, propitiating the inclusion of the rush, average load and idleness hours in the power system. It is worth mentioning that net PLC was used exclusively for the experiment, not having, therefore, any competition. With 
this, the load periods experienced by the system are directly related with the interference generated from the equipments connected to the power system, from the LPRAD as well as the LEEC as a whole.

This affirmation can be verified in Figure 2, which presents the throughput graph for one day of monitoring. It is observed that in the interval from $00: 00 \mathrm{~h}$ to $10: 00 \mathrm{~h}$ the throughput has a constant behavior, characterizing a period of small presence of noises. However, the interval from $10: 00 \mathrm{~h}$ to $14: 00 \mathrm{~h}$ presents a great variation in the throughput, which increases in the period from 15:00h to $17: 00 \mathrm{~h}$, period when the use of the power system is in its fullness.

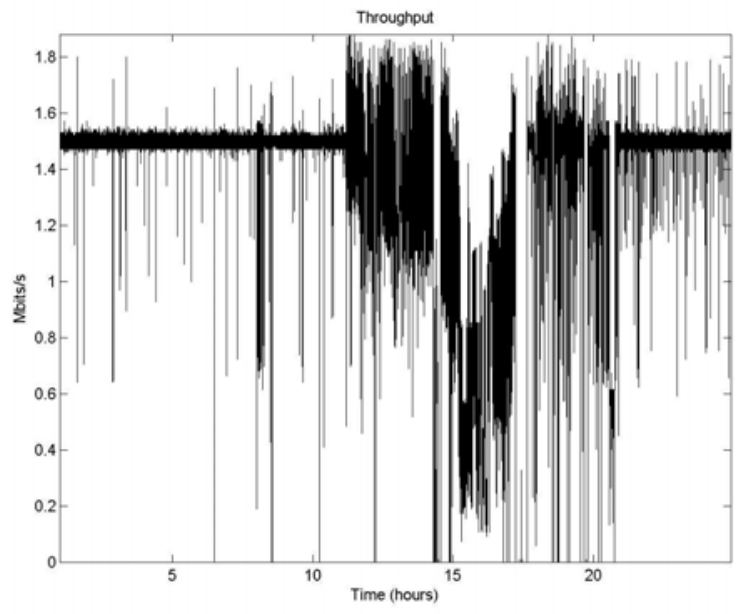

Fig. 2. Throughput of $24 \mathrm{~h}$ of observation

Table 1 contains the average values of the throughput, jitter and blocking probability obtained in the observation period.

TABLE I

OBTAINED VALUES - 24H OF OBSERVATION

\begin{tabular}{|c|c|}
\hline \multicolumn{2}{|c|}{ Observed Values } \\
\hline Throughput & $1.36264 \mathrm{Mbps}$ \\
\hline Jitter & $0.00407 \mathrm{~ms}$ \\
\hline Blocking Probability & $9.1066 \%$ \\
\hline
\end{tabular}

Based on the values observed in the prototype, a simulation model was constructed for the NS-2. The simulator allows error models to be created, this way, specific characteristics of the given network can be inserted, in what refers to the loss of packages. This is particularly interesting in the ratio where the physical characteristics of PLC network can be inserted in the model. With this, a more complete analysis can be made, enclosing from the inferior to the highest layers.

The error model uses basically two parameters: the percentage of packages loss and its probability distribution.

After the simulation, the values presented on Table 2 were obtained.

TABLE II

OBTAINED VALUES - MODEL SIMULATION

\begin{tabular}{|c|c|}
\hline \multicolumn{2}{|c|}{ Observed Values } \\
\hline Throughput & $1.3695 \mathrm{Mbps}$ \\
\hline Blocking Probability & $8.7745 \%$ \\
\hline
\end{tabular}

Therefore, from the relation of the simulation results with those achieved by the experimentation, it was possible to validate the model, inside of the simulation scenario established for the experiment. It was considered a confidence interval of $95 \%$, warm-up of $10 \%$ and reproduced the experiments at least 30 times for each performance measure.

\section{CASE STUDY: A CTR APPLICATION IN PARÁ}

In order to verify the feasibility of the PLC technology for digital inclusion purposes, a fictitious example based on an idea of a Regional Training Center (CTR), located in key cities of Pará. In these cities will be concentrate all the efforts to training teachers, community people, health agents and so on, of neighboring cities. These kinds of actions are common in Amazon due its particular characteristics.

A CTR is composed by three computer laboratories, each one with fourteen computers, two classrooms with one computer connected to Internet and the administration area.

The classrooms $\mathrm{A}$ and $\mathrm{B}$ are destined to video conferencing lectures; and the A, B and C laboratories are destined to internet access and distance learning classes.

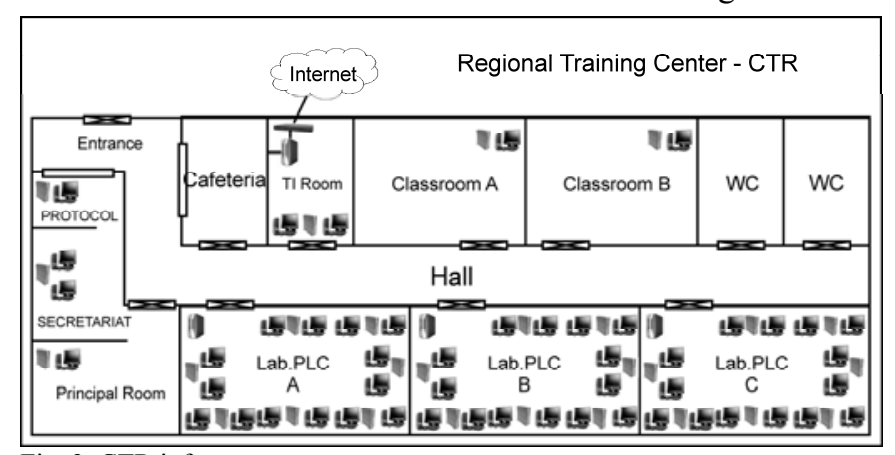

Fig. 3. CTR infrastructure

Figure 3 shows a PLC based infrastructure of a CTR.

Two scenarios were proposed for scalability system analysis: (a) one computer on laboratory A is running video streaming application, and the other ones are running typical HTTP applications over Ethernet; (b) the second case is similar to the first one, but running over PLC network.

Some simulations were performed considering video streaming application using H.263 standard, with rates of 16Kbps, $64 \mathrm{Kbps}, 256 \mathrm{Kbps}$ and VBR (Variable Bit Rate), with transmission based on Real Time Streaming Protocol (RTSP) [14].

To used model, the input data set was obtained from a trace file that had the main features of a streaming video transmission available in [15].

For a best view, an interval of 10 minutes was considered. Figures 4 to 7 present a comparison between Ethernet and PLC nets, considering the performance measures: throughput and blocking probability (this measurement was considered irrelevant to the Ethernet experiment).

To the simulation for $16 \mathrm{Kbps}$ were obtained the following values (Figure 4):

Blocking probability (PLC): 0.084203

Mean throughput for PLC: $0.01460423 \mathrm{Mbps}$

Mean throughput for Ethernet: 0.01502955 Mbps 

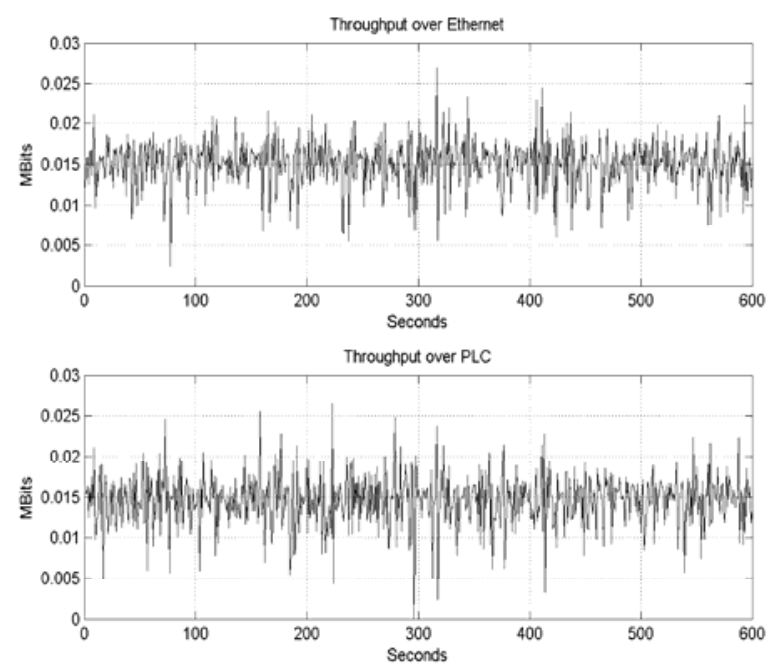

Fig. 4. Throughput for $16 \mathrm{Kbps}$

To the simulation for $64 \mathrm{Kbps}$ were obtained the following values (Figure 5):

Blocking probability (PLC): 0.087457

Mean throughput for PLC: $0.05840553 \mathrm{Mbps}$

Mean throughput for Ethernet: $0.05866267 \mathrm{Mbps}$
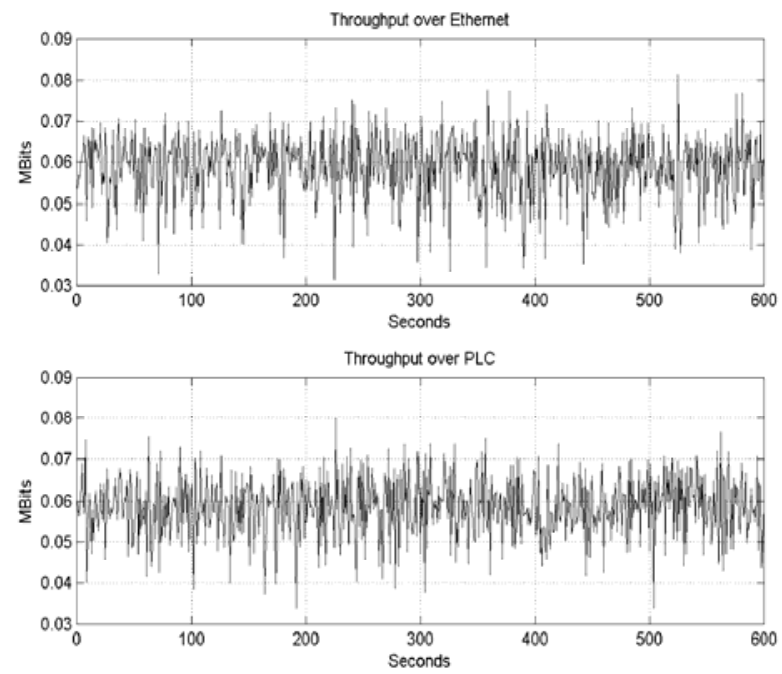

Fig. 5. Throughput for 64Kbps

To the simulation for $256 \mathrm{Kbps}$ were obtained the following values (Figure 6):

Blocking probability (PLC): 0.085939

Mean throughput for PLC: $0.2338981 \mathrm{Mbps}$

Mean throughput for Ethernet: $0.2044518 \mathrm{Mbps}$
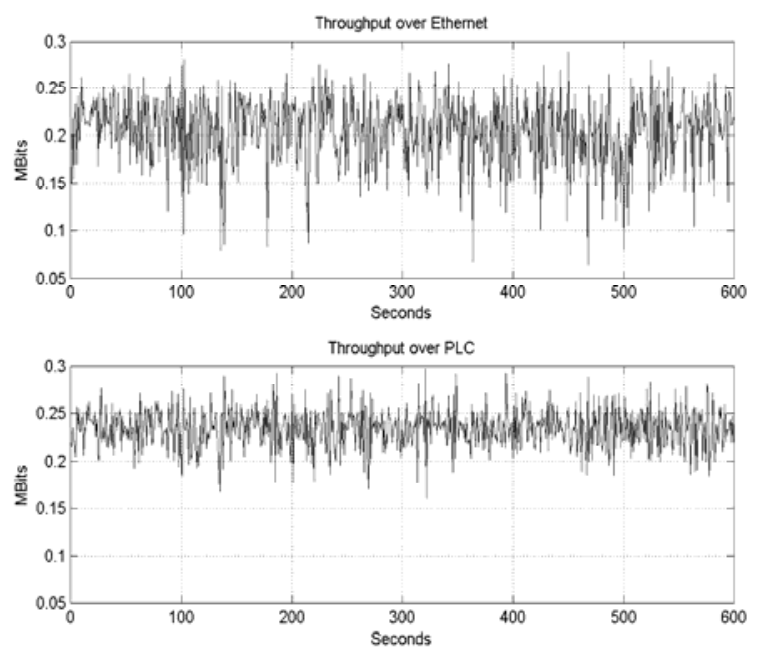

Fig. 6. Throughput for $256 \mathrm{Kbps}$

To the simulation for VBR were obtained the following values (Figure 7):

Blocking probability (PLC): 0.086421

Mean throughput for PLC: $0.3981872 \mathrm{Mbps}$

Mean throughput for Ethernet: 0. $3995093 \mathrm{Mbps}$
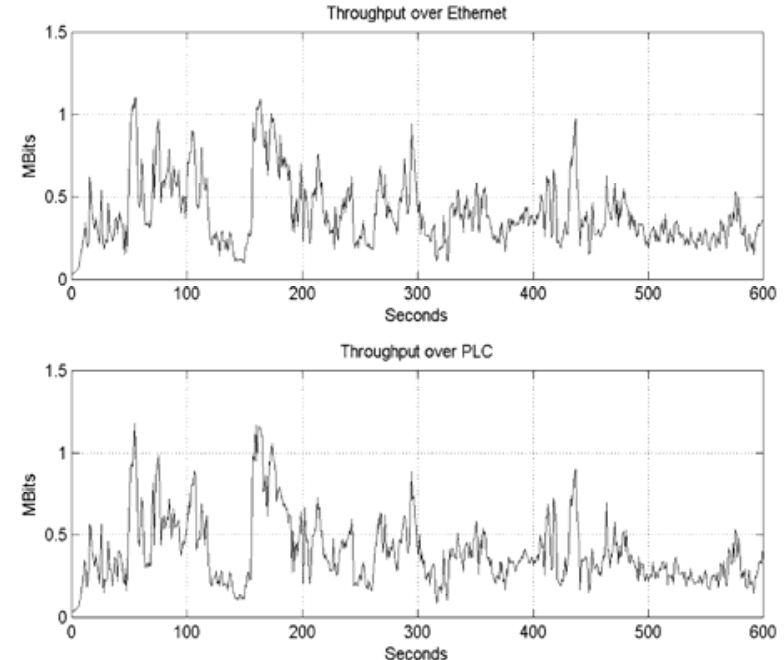

Fig. 7. Throughput for VBR

Although the PLC network presents a certain loss of packages due to the noise in the line, the transmission rates were satisfactory, not causing a significant degradation of the transmission in relation the Ethernet networks.

\section{CONCLUSIONS}

Observing the simulation results, it is possible to conclude that has a great viability in the use of PLC technology for action of digital inclusion in similar scenarios to the presented one in this paper, that basically characterize typical scenarios of the Amazon region. However, it is necessary to evaluate which applications will be used in the network; therefore an increase in the system's load can compromise the quality of the diverse applications that are running in the network. This paper is derived from a research line that investigates the viability of certain technologies under specific and singular conditions of the 
Amazon. For the factors presented in this paper and other diverse experiments carried out, it was observed that PLC technology has a great potentiality to provide access in regions with "poor" telecommunication infrastructure, due to, for instance: capillarity and peculiar bandwidth. As continuation of this research, it is being currently implemented in the LPRAD-UFPA an NS-2 module, PLC_NS, that incorporates characteristics of the environment to the traditional package routing evaluation made by that simulator. Moreover, a prototype connecting UFPA to Eletronorte, in medium voltage, is in final phase of implantation. This prototype will add an important quality to the feasibility studies carried out by this research.

\section{ACKNOWLEDGMENT}

Authors thank to Dr. Solon Carvalho for allowing the Stochastic Modeling Software (MODESTO) [16] use.

\section{REFERENCES}

[1] Andreou, G. T., Manitsas, E. K., Labridis D. P., et al (2003) "Finite element characterisation of LV power distribution lines for high frequency communications signals", In: $7^{\circ}$ International Symposium on Power-Line Communication and its Applications (ISPLC), Kyoto, Japão, 2003.

[2] Coutinho, M. M.; Francês, C. R. L.; Costa, J. C. W. A (2005). A Flexible Framework Proposal for the Return Path in Brazilian Digital Television. INFOCOMP - Journal of Computer Science, v. 4, n. 3, p. 42-49, 2005.

[3] Frahia, S. G. C; Rodrigues, J. C.; Gomes, H.; Cavalcante, G. P. S (2005). An Empirical Model for Propagation Loss Prediction in Indoor Mobile Communications Using Pade Approximant. Microwave and Optical Technology Letters, USA, 2005 (accepted November 2005, Article in Press).

[4] Freire, A. P. B.; Cavalcante, G. P. S (2005). Modelagem Híbrida de Canal para Sistemas Móveis em Área Suburbana Densamente Arborizada. In: XXI Simpósio Brasileiro de Telecomunicações - SBT 05, Campinas - SP, 2005.

[5] Hooijen, O. (1998) "A channel model for the residential power circuit used as a digital communications medium" In: IEEE Transactions on Electromagnetic Compatibility, Vol. 40, No. 4, November 1998.

[6] Hrasnica, H., Haidine, A., Lehnert, R. (2004) "Broadband Powerline Communications Networks - Network Design”, John Wiley \& Sons, Inc, 2004.

[7] Ma, Y. H., So, P. L., Gunawan, E. (2005) "Performance analysis of OFDM systems for broadband power line communications under impulsive noise and multipath effects" In: IEEE Transactions, Vol. 20, No. 2, Abril 2005.

[8] Meng, H. e Guan, Y. L. (2005) "Modeling and Analysis of Noise Effects on Broadband Power-Line Communications", In: IEEE Transactions on Power Delivery, Vol. 20, No. 2, Abril 2005.

[9] Ribeiro, A.R. L.; Francês, C. R. L.; Costa, J. C. W. A (2005), SensorBus: A Middleware Model for Wireless Sensor Networks. In: IFIP/ACM Latin America Networking Conference, 2005, Cali. Proceedings of the IFIP/ACM Latin America Networking Conference, 2005.

[10] Sanches, M. A. R.; Cavalcante, G. P. S.; Oliveira, R. A. N. (1999). Mobile Radio Propagation along Mixed Paths in Forest Invironment. Journal of Microwaves and Optoelectronics, UNB, v. 4, p. 42-52, 1999.

[11] The NS Manual. Available: http://www.isi.edu/nsnam/ns/.

[12] Zimmermann, M., Dostert, K. (2002) "Analysis and Modeling of Impulsive Noise in Broad-Band Powerline Communications", In: IEEE Transactions on Electromagnetic Compatibility, Vol. 44, No. 1 Fevereiro 2002.

[13] Zimmermann, M., Dostert, K. (2000) "The low voltage power distribution network as last mile access network-signal propagation and noise scenario in the HF-range" In: AEÜ Int. J. Electron. Communications, Vol. 54, No. 1, 2000.

[14] RTSP (Real-Time Streaming Protocol) - Available: http://www.ietf.org/rfc/rfc2326.txt.
[15] MPEG-4 and H.263 Video Traces for Network Performance Evaluation - $\quad$ Available: http://www.tkn.tuberlin.de/research/trace/trace.html

[16] Frances, C., Oliveira, E., Costa, J., Santana, M., Santana, R., Bruschi, S., Vijaykumar, N., Carvalho, S.: Performance Evaluation based on System Modelling using Statecharts exten-sions. Simulation Practice and Theory, Vol. 13, n. 7 (2005) 584-618. 